\title{
Estratégias de aprendizagem e aprendizagem virtual no ensino de Biologia
}

\author{
Learning and virtual learning strategies in Biology teaching
}

\author{
Joedh dos Santos*, Aldicea Craveiro de Lima Ferreira** e***, Alysson Brhian de Souza Muniz Silva*, Tacildo de \\ Souza Araújo*, Maria Erinete Reis Vila-Boas* e Luzia Mara dos Santos*** \\ *Instituto Federal de Educação Ciência e Tecnologia do Amazonas-IFAM, **Universidad de León-UNILEÓN-ES, ***Núcleo \\ de Estudos e Pesquisas em Psicopedagogia Diferencial da Universidade Federal do Amazonas-NEPPD-UFAM
}

\begin{abstract}
Resumo
O trabalho teve como finalidade a abordagem da Estratégia de aprendizagem para o aprender à aprender no ensino de Biologia e demais Ciências, enfocando à compreensão das fases básicas de desenvolvimento e ensino das estratégias de aprendizagem das novas de relação próxima e bem representada pela escola presencial e um novo paradigma, o sistema virtual de aprendizagem, sendo um grande suporte da Educação a Distância que com sua especificidade rápida e prática vem ganhando grande espaço no ensino. Utilizou-se a analítica qualitativa pela pesquis a ser uma avaliação completa de informações disponíveis que possibilitaram a compreensão do fenômeno deste trabalho.

Palavras-chave: Estratégias, aprendizagem, virtual, Biologia.
\end{abstract}

\begin{abstract}
The work aimed to approach the strategy of learning to learn to learn in teaching biology and other sciences, focusing on the understanding of the basic stages of development and teaching of new learning strategies of the close relationship and well represented by school and classroom a new paradigm, the virtual learning system, being a great support of the Distance Education that with its rapid and specific practice has gained great space education. We used the qualitative analytical by research to be a thorough evaluation of available information that enabled the understanding of this work phenomenon.

Keywords: Strategies, learning, virtual, Biology.
\end{abstract}

\section{Marco Teórico}

A aprendizagem acontece por meio da internalização, a partir de um processo anterior, de troca ${ }_{2}$ que possuiuma dimensão coletiva. Segundo VIGOTSKY (2008), o ato do aprender determina vários processos internos de desenvolvimento mental, que tomam corpo somente quando o sujeito que es tá a aprender interage com objetos em cooperação. Uma vez internalizados, esses processos tornam-se parte das aquisições do desenvolvimento cognitivo, mas esse sistema, na prática, faz-se principalmente na descoberta das rotas eficazes individuais para o aprender e, de estratégias de aprendizagem.
O aluno apresenta sérias limitações para internalizar e compreender o conteúdo do conhecimento, do ensino principalmente na área da Biologia. Muitas dificuldades neste ensino são: A confusão entre a natureza da Ciência e os processos da Ciência. Um ensino baseado em procedimentos não implica necessariamente uma boa compreensão da natureza da Ciência (EICK, 2010). Outro fator é o desconhecimento dos enfoques didáticos eficazes para o ensino de Biologia, procedente do escasso domínio do didático-pedagógico e estratégias de aprendizagem significativas a hora de evidenciar um ensino explicito e reflexivo da natureza da Ciência Biologia (ACEVEDO, 2007). Por último e não pouco importante, a falta de conhecimento metodológico profundo de nível científico. Um profess or deve propiciar uma boa compreensão dos aspectos científicos com os vínculos entre Ciência, cidadania e valores (QUINTANILLA, 2006).

A maior parte dos alunos sabe ou aprende como processar a informação e desenvolver uma estratégia de aprendizagem ou um plano organizado quando confrontado com um problema social, acadêmico ou relacionado com um emprego. Contudo, outros consideram que este processo cognitivo é muito difícil. Leem e releem informação sobre este assunto, mas não conseguem reter as ideias principais. Têm um bom vocabulário oral, mas os relatórios orais e escritos são simplistas e aborrecidos. Podem estudar durante horas para um teste, mas o resultado não vai de encontro às suas expectativas nem às do professor, o desenvolvimento cognitivo está além de simples procedimentos, é necessário um investimento substancial no ensino, compreensão e domínio das estratégias. Esse processo de desenvolvimento cognitivo está centrado justamente na possibilidade de o sujeito ser, constantemente, colocado em situações problema que provoquem a construção de conhecimentos e conceitos, a partir da zona de desenvolvimento proximal. Ou seja, o sujeito necessita usar os conhecimentos já consolidados, desestabilizados por novas informações, que serão processadas, colocadas em relação com outros conhecimentos, de outros sujeitos, num processo de interação, para só então, serem consolidadas como um conhecimento novo. Inúmeras investigações defendem que uma das maiores diferenças entre os estudantes eficientes e os ineficientes é a sua compreensão e o uso de boas estratégias de aprendizagem. DEHSLER E LENZ (1989) definem uma estratégia como o "modo como o aluno aborda uma 
tarefa: inclui o modo como uma pessoa atua quando planifica, executa e avalia a realização de uma tarefa e os seus resultados".

Tem sido proposto e investigado um grande número de modelos experimentais de estratégias, inclusivamente treino estratégico acadêmico, aprendizagem recíproca, e, em nível mais específico, as estratégias de aprendizagem podem ser consideradas como qualquer procedimento adotado para a realização de uma determinada tarefa (Da Silva \& Sá, 1997 in IESDE, 2003).

Ainda que haja diferenças de modelos, todas convergem em dois pontos: $O$ inicial é o grande número de estudantes, muitas vezes designados como "de risco", desmotivados, imaturos, com dificuldades de aprendizagem, etc. que são deficientes no uso de estratégias de aprendizagem. Estes estudantes podem-se encontrar tanto em programas de educação regular como em educação especial. Outro ponto é estes estudantes poderem aprender estratégias de aprendizagem o que os vai ajudar a abordar as tarefas com mais eficácia e eficiência, aumentando as suas hipóteses de sucesso. Tudo depende mesmo é do processamento das informações que em seguida torna-se aprendizagem, consequentemente possibilidades de solução de problemas.

Uma classificação de estratégias de aprendizagem, evidenciadas através de etapas de processamento da informação, que se verá mais adiante é baseada em Jesus Beltrán, Director del Departamento de Psicología Evolutiva y de la Educación de la Universidad Complutense de Madrid por BÉLTRAN (2002), onde apresenta uma abordagem do assunto considerando estratégias e tipo de avaliação possíveis na Educação a Distância, em uma perspectiva sócio - interacionista.

Antes de mencionar a aprendizagem virtual, é importante ressaltar sobre a educação à distância. GARCÍA (2013) afirma que é difícil encontraruma definição certa quanto a Educação a Distância, apesar de tantas denominações, estruturas, metodologias, organização, o uso de tecnologias, tamanho da instituição, demandas diferenciadas e projetos, todos os sistemas de ensino a distância tem em comum uma série de características em comum conhecidos por vários estudiosos, um exemplo a seguir, (1982, ARETIO, p.14): O término da educação a distância cumpre um amplo aspecto de diversas formas de estudo e estratégias educativas, que tem em comum o feito de que elas não se cumprem mediante a continuidade tradicional física continua de professores e alunos em locais especiais para fins educativos: Esta nova forma educativa inclui todos os métodos de ensino em que devido à separação existente entre alunos e professores, as fases interativas ensino proativa são conduzidas mediante a palavra impressa, ou elementos mecânicos e eletrônicos.

\section{Aprendizagem Virtual: uma visão sócio- interacionista.}

Para BARBERÀ, ROMISZOWSKI, SANGRÀ ET. AL (2006), a palavra distância pode ter muitos significados. Primeiro que pode seruma distância geográfica, distância de tempo e possivelmente inclusa na distância intelectual.
Segundo, o termo educação a distância pode seraplicado a uma variedade enorme de programas que dão serviços a numerosas informações utilizando uma larga variedade de meios. Alguns utilizam material impresso, outros, telecomunicações e muitos utilizam os dois. Finalmente, essas mudanças na tecnologia desafiam as formas tradicionais que definem a educação à distância.

A aprendizagem a partir dos ambientes virtuais, que segundo elemento constituinte de uma proposta sócio interacionista de educação à distância com uso da informática ou EADI, permite incentivar a reflexão crítica e a possibilidade de escolhas; a Internet abre um novo horizonte e cria novos paradigmas para a discussão, na medida em que permite a comunicação, por intermédio de ferramentas síncronas ou assíncronas. A mediação pedagógica deve utilizar-se, obrigatoriamente, dessas ferramentas. A construção de ambientes virtuais de aprendizagem passa por etapas que definem e desenham sua aplicabilidade, suas possibilidades de sucesso ou de fracasso.

PALOFF (2002) ajuda a refletir sobre a condição de realidade mostrando a situação em que, a definição da concepção de educação a ser oferecida é determinante do processo. A escolha das ferramentas de interação, a arquitetura da plataforma e do administrador de sistemas depende dessa escolha. As ferramentas de interação multidirecionais, serão prioridade em uma proposta de curso sócio-interacionista, por exemplo. Em uma proposta de instrução programada, fundamentada na mera transmissão de conteúdos, bastariam ferramentas como os e-mails, listas e perguntas frequentes, onde apenas os alunos perguntam e os tutores respondem. Outro ponto importante é a escolha da tecnologia adequada à realidade dos atores envolvidos no processo com a coerência na escolha de um recurso que esteja disponível e ao alcance de alunos e professores, tanto do ponto de vista técnico, quanto do financeiro.

SEGUNDO SILVA (2003, p. 55) - o suporte digital a pluralidade significante é dada como dispositivo material: o sujeito (aluno, pesquisador), não apenas interpreta mais ou menos livremente como também organiza e estrutura, ao nível mesmo da produção, o que é uma estratégia extremamente delicada de saber-se lidar e controlar sem o risco de entrar num mundo de fantasia de base na ideia de terceiros, onde tudo não pass a da mera reprodução alienada, sem critica e autenticidade própria. É necessário saber fazer uso do conjunto enorme de mídias, a imagem dinâmica - perfeita, em CD ou DVD, a fala em tempo real, a troca, de acordo com o momento e a necessidade de cada um, através do e-mail, do chat, do fórum e das lis tas de discussão, nummovimento contínuo e múltiplo que atinge a todos os elementos envolvidos na aprendizagem - enquanto suporte, às estratégias mentais de aprendizagem. A Internet concretiza a possibilidade de apropriação e de personalização da mensagem recebida, da reciprocidade da comunicação na virtualidade. Por meio de fóruns, de listas de discussões, de salas de aula virtuais, de espaços para realização de trabalhos em grupo e de espaços de pesquisa compartilhada, incentiva-se a troca de informações entre os alunos, provocando a socialização do conhecimento. 


\section{Avaliar no sistema virtual.}

Vendo-se a aprendizagem como um fenômeno que ocorre no espaço relacional e dialógico com o outro, é necessário que a avaliação seja suficientemente abrangente considerando as pectos, como:

Funções cognitivas e aspectos afetivos (valores, atitudes, emoções) e os níveis de conhecimento (potencial e real). A visão do process o segundo o professor, o que ele des eja ensinar, suas expectativas e aspirações.

A interação que ocorre entre o aluno e o professor : modalidades, ritmos, recursos mediadores, a ação sobre as descobertas do estudante e do professor,já que ambos aprendem neste processo.

O processo ensino-aprendizagem: como um todo, incluindo o seu "envoltório" sócio-histórico (meta avaliação). VALDÉS (2002) faz refletir na seguinte ideia: Propõe-se que as novas tecnologias da informação, em conjunto com outras mudanças sociais e culturais estão dando origem à nova cultura de aprendizagem que se caracteriza por encontrar a s ociedade de informação, o conhecimento múltiplo e aprendizagem contínua. Isto significa que o fornecimento de informações não é o suficiente, no entanto, é necessário ensinar os alunos a pesquisar, organizar e interpretar. (p. 139). Nessa perspectiva, a avaliação será ponto principal a ser repensado e retrabalhado sob a possibilidade de permitir que em real valor para que aconteça a aprendizagem seja preciso, a transposição dos métodos e técnicas tradicionais para novos prismas de atividades que levem o aprendiz a tomar em suas mãos suas responsabilidades globais de aprender com significado, mas sabendo o que aprende, por que aprender, e, para quê, aprende.

\section{O Ensino de Estratégias.}

Os vários modelos de ensino de estratégias visam substituir uma aprendizagem ineficiente e pouco efetiva por estratégias que levem ao sucesso e a um nível mais alto de realização do sujeito que aprende. Alguns dos princípios defendidos por especialistas estão aqui resumidos. (V. DESHLER AND SCHUMACHER 1986; SWANSON 2001).

Exigir a participação dos alunos: Este aspecto é um dos mais importantes da instrução de estratégias de aprendizagem. Além da aplicação de técnicas de motivação, é fundamental fazer com que o estudante veja como a estratégia o vai ajudar em problemas específicos.

Identificar e ensinar os pré-requisitos: $O$ ensino de estratégias ocorre em qualquer idade (contar pelos dedos é uma estratégia universal para crianças de 2 ou 3 anos), mas muitas estratégias exigem o conhecimento das aprendizagens básicas. A estratégia de escrever uma frase, a possibilidade de um rendimento com sucesso ocorrerá se os alunos souberemidentificar substantivos, verbos e preposições.

Aprender a estratégia: $O$ professor primeiramente deverá ter total domínio da estratégia por forma a fazer de modelo, levando então, os alunos a ver os passos dados de um modo natural e confortável. O domínio de estratégias permite ao professor manter o enfoque na instrução dos alunos. E quanto, a avaliação, relativa à aprendizagem à distância, pode e deve ter as mesmas funções da avaliação realizada em aprendizagens presenciais. E esta avaliação, sob um enfoque sóciointeracionista, por sua vez, deve possuir algumas qualidades, tais como: $1-\mathrm{E}$ nfoque pedagógico consistente e coerente com a proposta do curso desenvolvido; 2 - Explicitação de metas, critérios, padrões; Indicadoras de possibilidades de interdisciplinaridade; 3 - Grau facilitador da estrutura avaliativa, entre a autogestão do conhecimento e a diretividade do processo de ensino-aprendizagem; 4 Característica formativa (de acompanhamento do processo), suficiente e oportunamente realizada, e conjugada com as características diagnósticas e somativa do processo; e, 5 - Consciência do contexto de aprendizagem, em interação com as características do sujeito que aprende.

\section{Classificação das Estratégias de Aprendizagem e Avaliação.}

BELTRÁN (1993) apresenta um significante estudo e a classificação das estratégias de Aprendizagem (Tabela-I) que leva a considerar a importância do estudo e domínio dessa variável no seguimento cientifico da educação. Assim, o professor deve trabalhar e ter a prática da construção de um "desenho" ou "mapa" da avaliação que pretende proceder, envolvendo a análise do contexto ou realidade em que a avaliação irá acontecer; o "recorte" dos aspectos a seremavaliados; a elaboração, aplicação e avaliação das estratégias avaliativas; o tratamento a ser dado aos resultados obtidos.

Tabela 1.

Estratégias de Aprendizagem e situações aplicáveis na Educação a Distancia.

\begin{tabular}{c|l}
\hline $\begin{array}{c}\text { ES TRATEGIAS DE } \\
\text { APRENDIZAGEM }\end{array}$ & \multicolumn{1}{|c}{$\begin{array}{c}\text { PROCESSOS COGNITIVO - } \\
\text { EMOCIONAIS }\end{array}$} \\
\hline Sensibilização & $\begin{array}{l}\text { Motivação, formação de atitudes, } \\
\text { controle emocional, autoestima. }\end{array}$ \\
\hline Atenção & $\begin{array}{l}\text { Atenção (global ou seletiva e fixada } \\
\text { ou meta atenção). }\end{array}$ \\
\hline Aquisição & $\begin{array}{l}\text { Compreensão, retenção, } \\
\text { transformação. }\end{array}$ \\
\hline Personalização & $\begin{array}{l}\text { Criatividade, pensamento crítico, } \\
\text { autoregulação (planificação, } \\
\text { regulação, avaliação). }\end{array}$ \\
\hline Recuperação & $\begin{array}{l}\text { Primárias (relação entre o aluno e o } \\
\text { material) e Secundárias (entre o } \\
\text { aluno e o ambiente de trabalho em } \\
\text { geral). }\end{array}$ \\
\hline
\end{tabular}

A tabela a seguir (Tabela-2), as socia as estratégias de aprendizagem descritas por Beltrán a algumas possibilidades de avaliação, viabilizáveis na Educação a Distância. 
Tabela 2.

Associação das estratégias de aprendizagem a avaliação

\begin{tabular}{|c|c|}
\hline $\begin{array}{c}\text { ESTRATÉGIAS } \\
\text { DE } \\
\text { APRENDIZAGEM }\end{array}$ & $\begin{array}{c}\text { SITUAÇÕES AVALIATIVAS } \\
\text { APLICÁVEIS À EDUCAÇÃO } \\
\text { A DISTÂNCIA }\end{array}$ \\
\hline Sensibilização & $\begin{array}{l}\text { Exercícios diagnósticos, utilizados } \\
\text { no início do curso, verificando os } \\
\text { conhecimentos prévios e o espectro } \\
\text { motivacional do aluno. Pode-se } \\
\text { incluir a resposta a escalas de } \\
\text { atitudes, focalizando em especial as } \\
\text { que são esperadas no curso e a } \\
\text { construção de um portfólio, durante } \\
\text { o mesmo. } \\
\text { Atividades autoavaliativas } \\
\text { realizadas ao final de cada unidade } \\
\text { programática do curso. } \\
\text { Atividades que necessitem de } \\
\text { controle emocional, como debates } \\
\text { virtuais. }\end{array}$ \\
\hline Atenção & $\begin{array}{l}\text { Atividades que exijam percepção } \\
\text { de detalhes, respostas rápidas, } \\
\text { autocorreção de exercícios, } \\
\text { consulta a bibliotecas virtuais. }\end{array}$ \\
\hline Aquisição & $\begin{array}{l}\text { Verificação dos conceitos } \\
\text { construídos, através das avaliações } \\
\text { presenciais e a distância, utilizando } \\
\text { todas as ferramentas de interação } \\
\text { (FIs) disponíveis. Nunca é demais } \\
\text { ressaltar que as avaliações devem } \\
\text { sempre transcender as situações em } \\
\text { que os conteúdos foram abordados, } \\
\text { permitindo a transposição dos } \\
\text { conteúdos a outras situações - } \\
\text { problema. }\end{array}$ \\
\hline Personalização & $\begin{array}{l}\text { Atividades avaliativas propostas } \\
\text { pelos alunos, avaliação dos } \\
\text { próprios resultados e das avaliações } \\
\text { realizadas. Participação em listas } \\
\text { de discussão e fóruns, participação } \\
\text { de "conversas" realizadas no "café } \\
\text { virtual", que permitem uma } \\
\text { abrangência maior, transcendente } \\
\text { aos conteúdos abordados nas aulas. }\end{array}$ \\
\hline Recuperação & $\begin{array}{l}\text { Atividades de revisão dos } \\
\text { conteúdos trabalhados no curso, } \\
\text { trabalhos de grupo, avaliação das } \\
\text { dúvidas apresentadas na tutoria on } \\
\text { line e presencial. Avaliações } \\
\text { propostas ao final de cada aula, em } \\
\text { relação ao conteúdo da mesma. }\end{array}$ \\
\hline
\end{tabular}

Reconhecer e recompensar o esforço do aluno: Os alunos com problemas de aprendizagem muitas das vezes têm uma história de insucesso. O professor deve ser sensível a isso e valorizar de varias maneiras todo o esforço do aluno, seja com um elogio ou dar um feedback positivo para realizações ainda que modestas.

Levar o estudante a ter o domínio de estratégias: Os alunos devem aprender as suas estratégias a um nível que lhes permita o seu uso automático. As investigações indicam que sem este nível de realização, muito pouco será generalizado a uma aplicação real. Deixem os alunos terem os seus próprios quadros para apontar o seu progresso na aprendizagem de uma estratégia. Contudo, o professor deve estabelecer o nível de realização. Com algumas estratégias não será razoável estabelecer os 100\% como nível de realização.

Instrução integrada: Embora haja várias etapas na aquisição do domínio de uma estratégia de aprendizagem, elas não são sempre lineares nem sequenciais. Por exemplo: a capacidade de generalizar é geralmente o último passo, mas pode ser introduzida em qualquer altura, especialmente se referida por um aluno. O objetivo último é conseguirque os alunos incorporem as estratégias no seu sistema de informação.

Dar explicações diretas: Os professores devemrealçar o processo cognitivo envolvido na aplicação de estratégias de aprendizagem. Para que os alunos aprendam as etapas de uma estratégia particular, eles deverão usar imagens visuais, formular hipóteses, ou relacionar a nova informação com conhecimentos anteriores. O profess or deve demonstrar e encorajar estes processos cognitivos e metacognitivos.

Promover a generalização: $\mathrm{O}$ uso de estratégias terá relevância nas situações de aprendizagem dos alunos somente se estes souberemutiliza-las em várias situações de aprendizagem tendo completo domínio dos seus passos, assim, conseguindo generalizar o seu uso. Os professores devem seguir e reforçar o uso de estratégias de aprendizagem sempre que surja oportunidade para isso. E os pais deveriam ser encorajados a fazer o mesmo em casa.

Encorajar a adaptação e o desenvolvimento: Toda estratégia aprendida e generalizada, tornar-se parte funcional do processo mentaldo aluno. As modificações, para adaptar fatores temporais, conteúdo, ou situações ambientais, são apropriadas. O objetivo último é conseguir que os alunos compreendam todo o processo de aquisição de estratégias de aprendizagem, sendo capazes de desenvolveras suas próprias.

\section{Discussões}

Pensar estratégias de aprendizagem e aprendizagem virtual no ensino de Biologia nos possibilitou um estudo enriquecedor para nossa formação enquanto acadêmico de Biologia, mas também, nos fez sentir na pele, o descaso das autoridades quanto a qualidade de ensino que, por sua vez, obrigatoriamente deveria estar pautada em marcos científicos que imprimam responsabilidades educacionais visíveis e executáveis em políticas públicas da Educação, e que, acompanhe os movimentos e transformações constantes que se impõem a educação bancaria, apenas voltada para o mercado de trabalho segundo a demanda do capital, ou seja, do modelo econômico capitalista que por si só já aniquila com a criatividade e o potencial inovador e criador do/a aluno/a. 


\section{Conclus ões}

Diante de todo apelo virtual que hoje determina a e orienta a vida de milhões de pessoas que estudam fazendo uso das novas tecnologias, o governo brasileiro de primar urgente pela ruptura do continuísmo do discurso hipócrita dos políticos mal intencionados que se envolvem com a Educação nos altos escalões do poder federal, estaduale municipal. Muitos deles sem nenhuma capacidade técnica-profissional para lidar com rotas educativas que responsáveis porvidas, sonhos e potencial humano - trans formando o segmento educativo, em mera máquina reprodutora de modelos importados, que, de nada valem à realidade brasileira - nos impondo assim, enquanto estudantes da Educação Básica e do Ensino Superior - no nosso caso Licenciatura em Biologia -, a meras cópias de programas falhos e retrógrados quanto à verdadeira missão da Educação segundo a UNESCO ensinar ao aluno se resume: em ensinar o/a aluno/a: a saber-fazer; ser; aprender e a conviver para vida e solução dos problemas que lhes acomete o convívio social e escolar, quiçá, familiar na sua plenitude invocando os valores e as possibilidades de uma vida equilibrada por todos ter oportunidades igualitárias. Hoje temos plena convicção que dominar as novas tecnologias da educação concomitante ao domínio das dificuldades contemporâneas de aprendizagem e, sobretudo, métodos, técnicas e estratégias de aprendizagem é imprescindível para uma boa atuação do campo da ensinagem das Ciências naturais, em especial da Biologia.

\section{Referencias}

ACEVEDO, J. Investigación cientifica, naturaleza de la ciencia y enseñanza de las ciencias (II). Revista Eureka sobre Enseñanza y Divulgación de las Ciencias, Cádiz, v. 4, n. 3, p. 571-579, 2007.

BÉLTRAN, Jesús L. et al. Procesos, estrategias y técnicas de aprendizaje. Madrid: Sintes is, 2002.

Deshler, D.D.,v\& Lenz, B.K. (1989). The strategies instruction approach. International Journal of Learning Disability, Development and Education.

GARCÍA, L. La educación a distancia: de la teoría a la práctica. 2013. Ed. 2. Pg. 14, 2013.

SCHUMAKER, J.B., DESHLER, D.D., \& ELLIS, E.S. (1986). Intervention issues related to the education of LD adolescents. In J.K. Torgeson \& B.Y.L. Wong (Eds.), Psychological and educational perspectives on learning disabilities (pp. 329-364). New York: Academic Press.
SCHUMAKER, J. B., \& DESHLER, D. D. (1992). Validation of learning strategy interventions for students with learning disabilities: Results of a programmatic research effort. In B. Y. L. Wong (Ed.), Contemporary intervention research in learning disabilities: An international perspective.

EICK, C. J. Inquiry, nature of science, and evolution: the need for a more complex pedagogical content knowledge in science teaching. Electronic Journal of Science Education, Georgetown, v. 4, n. 3, 2000.

PALINCSAR, A., \& Brown, A. (1984). Reciprocal teaching of comprehension-fostering and comprehension-monitoring activities. Cognition and Instruction, 1, 117-175.

PALOFF, Rena M. Construindo comunidades de aprendizagem no ciberespaço. 'Porto Alegre: Artmed, 2002.

QUINTANILLA, M. La Ciencia en La Escuela: Un saber fascinante para aprender a "leer el mundo". Pensamiento Educativo, Santiago de Chile, v. 39, n. 2, p. 177-204, 2006.

SILVA, Marco. EAD on - line, cibercultura e interatividade. In: ALVES, Lynn \& NOVA, Cristiane. Educação a distância: uma nova concepção de aprendizado e interatividade. Rio de Janeiro: Ed. Futura, 2003.

SWANSON, H. L. (2001). Research on intervention for adolescents with learning disabilities: A meta-analysis of outcomes related to high-order processing. The Elementary School Journal, 101, 331-348.

VALDÉS, María Teresa M. Estrategias de aprendizaje: Punto de encuentro entre la Psicología de la Educación y la Didáctica. In ROSA, Dalva E. G. \& SOUZA, Vanilton C. de. Didática e práticas de ensino: interfaces com diferentes saberes e lugares formativos. Rio de Janeiro/Brazil: DP\&A, 2002.

VYGOTSKY, L. S. Pensamento e Linguagem. São Paulo/Brazil: Editora Martins. Edição 04, 2008.

\section{Agradecimientos}

Pela graça a Deus, por todas as conquistas alcançadas. Aos pesquisadores da Universidade Federal do Amazonas - UFAM pela cooperação e auxílio no trabalho.

Aos pesquisadores do Instituto Federal de Educação, Ciência e Tecnologia do Amazonas - IFAM por ajudar na produção desse trabalho. 